\title{
Should High-dose Daptomycin be an Alternative Treatment Regimen for Enterococcal Endocarditis?
}

\author{
Maddalena Peghin (D) Alessandro Russo - Filippo Givone • \\ Martina Ingani · Elena Graziano · Matteo Bassetti
}

Received: June 17, 2019 / Published online: August 19, 2019

(C) The Author(s) 2019

\begin{abstract}
Introduction: Previous series on the use of daptomycin in enterococcal infective endocarditis (EIE) have shown various outcomes, including higher mortality rates. We analyzed the effectiveness of high-dose daptomycin for the treatment of EIE.

Methods: We performed a prospective study from 2010 to 2018 in a referral center in patients with native (NVE) and prosthetic valve endocarditis (PVE) due to Enterococcus spp. The standard high-dose daptomycin at our institution is $10-12 \mathrm{mg} / \mathrm{kg} /$ day (CLCr $>30 \mathrm{ml} / \mathrm{min}$ ). We compared the efficacy of a daptomycinbased regimen (DBR) versus daptomycin-sparing regimen (DSR) and daptomycin monotherapy versus combination therapy. Primary endpoints of the study were evaluation of risk
\end{abstract}

Enhanced digital features To view enhanced digital features for this article go to https://doi.org/10.6084/ m9.figshare.9165305.

M. Peghin $(\varangle)$ - A. Russo · F. Givone · M. Ingani ·

E. Graziano · M. Bassetti

Infectious Diseases Division, Department of

Medicine, University of Udine and Azienda Sanitaria

Universitaria Integrata di Udine, Udine, Italy

e-mail: maddalena.peghin@gmail.com

M. Bassetti

Department of Health Sciences, University of

Genoa, Genoa, Italy factors associated with 30-day mortality and failure at end of therapy.

Results: We collected 43 EIE cases; 29 were NVE (67.4\%). Overall, $16(37.2 \%)$ were treated with DBR, mainly with combination regimens (11, $68.7 \%)$, in the majority of cases in association with ß-lactam $(7,43.7 \%)$. The mean administered dose of daptomycin was $10.125 \mathrm{mg} /$ $\mathrm{kg} /$ day (range $8-12 \mathrm{mg} / \mathrm{kg} /$ day). Overall, patients treated with DBR compared with patients treated with DSR had no higher mortality rates and/or failure at end of therapy (6.2\% vs. $22.2 \% ; P 0.41$ and MICs $0.25-2 \mathrm{mg} / \mathrm{l}$, $6.2 \%$ vs. $3.7 \% ; P 1.0$ ). In the sub-group of patients with NVE and PVE treated with DBR and DSR, no difference was found regarding the primary endpoints on the single or combined use of daptomycin.

Conclusion: Our findings suggest that highdose daptomycin might be used as an alternative treatment regimen in EIE.

Keywords: Daptomycin; Enterococcal infective endocarditis; Enterococcus spp.; High-dose daptomycin

\section{INTRODUCTION}

Over the past decades, continuous changes in epidemiologic and clinical characteristics of infective endocarditis (IE) have shown that enterococci have emerged as the third most 
common pathogen (10-15\% of IE cases) following staphylococci and streptococci $[1,2]$.

Treatment of enterococcal IE (EIE) is known to be challenging, because enterococci have shown the ability to develop antibiotic resistance with increasing high-level aminoglycoside resistance (HLAR) and cure of IE usually requires a prolonged course of antibiotics (up to 6 weeks) with potential adverse events $[3,4]$. In addition to established antimicrobials (ampicillin plus ceftriaxone or ampicillin with aminoglycosides), old (teicoplanin, vancomycin) and novel antibiotics (dalbavancin) have being investigated to improve the effectiveness of antibacterial strategies [5-7].

Daptomycin is a cyclic lipopeptide antibiotic with highly concentration-dependent bactericidal activity against gram-positive bacteria and has been highly prescribed in the setting of resistant enterococci [8]. Daptomycin was approved for the treatment of right-sided endocarditis caused by Staphylococcus aureus at a dose of $6 \mathrm{mg} / \mathrm{kg}$, although clinical and microbiologic data support improved efficacy at higher doses [9]. In addition, several in vitro studies and some case reports have demonstrated synergistic activity against enterococci with the combination of daptomycin and other antibiotics $[10,11]$. Daptomycin at higher doses ranging from 8 to $14 \mathrm{mg} / \mathrm{kg} /$ day has been used in EIE with variable and conflicting results, including non-favorable microbiologic and clinical response [12-14].

We have integrated the use of high-dose (> $10 \mathrm{mg} / \mathrm{kg}$ ) daptomycin [15] in monotherapy or combination therapy in our treatment armamentarium for EIE. The aim of this study is to report the experience with daptomycin compared with the standard treatment option [3] at our institution.

\section{METHODS}

We performed a prospective 9-year study (2010-2018) at the Santa Maria Misericordia Hospital in Udine, a tertiary-care teaching hospital (1000 beds) that is a referral regional center for IE with a heart surgery department. All consecutive adult patients ( $>18$ years) with native (NVE) and prosthetic valve endocarditis (PVE) sustained by Enterococcus spp. were included.

IE was defined according to the Duke's modified criteria. Indication for surgery was based on ESC clinical guidelines [3].

Minimum inhibitory concentrations (MICs) were determined by use of either $E$ test or Sensititre. MIC $\geq 256 \mathrm{mg} / \mathrm{l}$ was recorded as HLAR and MIC $>4 \mathrm{mg} / \mathrm{l}$ as daptomycin non-susceptible, in accordance with the European Committee on Antimicrobial Susceptibility Testing (EUCAST) recommendations.

The standard recommended high dosage of daptomycin in EIE at our institution is $10-12 \mathrm{mg} / \mathrm{kg} /$ day $\quad(10-12 \mathrm{mg} / \mathrm{kg} / 48 \mathrm{~h}$ if $\mathrm{CLCr}<30 \mathrm{ml} / \mathrm{min}$, including hemodialysis and CAPD) infused over $15 \mathrm{~min}$ [15].

Primary endpoints of the study were evaluation of risk factors associated with 30-day mortality rates and with failure at end of therapy. We compared a daptomycin-based regimen (DBR) vs. daptomycin-sparing regimen (DSR) as a first-line or salvage therapy and daptomycin monotherapy versus daptomycin used in combination.

Patients were judged to have clinical failure if they had no response to the antimicrobial treatment based on ongoing fever, leukocytosis, other clinical parameters and/or relapse of IE. Treatment failure was defined as an inadequate response to antibiotic with persistence of fever and/or persistent bacteremia after 6 days of adequate therapy, requiring an antibiotic change.

Quantitative variables were reported as the mean ( \pm standard deviation), and qualitative variables were reported as number and percentage. Statistical analyses were performed with SPSS-PC+, version 22.0 (SPSS, Chicago, IL). The study was approved by the local institutional review board.

All procedures performed in studies involving human participants were in accordance with the ethical standards of the University of Udine and Azienda Sanitaria Universitaria Integrata di Udine (IRB NR 505) and with the 1964 Helsinki Declaration and its later amendments or comparable ethical standards. The need for informed consent was waived by the IRB because of the observational nature and minimal risk of the study. 
Table 1 Demographic features, comorbidities, characteristics and outcome of 43 episodes of EIE treated with DCR and DSR

\begin{tabular}{|c|c|c|c|c|}
\hline Variables & All, $n 43$ (\%) & DBR $n 16(\%)$ & DSR, $n 27$ (\%) & $\boldsymbol{P}$ \\
\hline Age (years), mean $+S D$ & $67.3 \pm 15.6$ & $67.1 \pm 15.7$ & $67.3 \pm 15.5$ & 0.81 \\
\hline Male & $29(67.4)$ & $10(62.5)$ & $19(70.4)$ & 0.73 \\
\hline \multicolumn{5}{|l|}{ Underlying condition } \\
\hline Diabetes & $8(18.6)$ & $6(37.5)$ & $2(7.4)$ & 0.04 \\
\hline Chronic renal failure & $15(34.8)$ & $6(37.5)$ & $9(33.3)$ & 1.0 \\
\hline Transplantation & $1(2.3)$ & $1(6.2)$ & 0 & 0.37 \\
\hline Neoplasm & $3(6.9)$ & $2(12.5)$ & $1(3.7)$ & 0.54 \\
\hline Liver cirrhosis & $1(2.3)$ & 0 & $1(3.7)$ & 0.31 \\
\hline COPD & $5(11.6)$ & $3(18.8)$ & $2(7.4)$ & 0.3 \\
\hline Modified Charlson score, median (range) & $4.4 \pm 2.5$ & $4.1 \pm 2.9$ & $4.5 \pm 2.4$ & 0.55 \\
\hline \multicolumn{5}{|l|}{ Type of IE } \\
\hline Native valve endocarditis & $29(67.4)$ & $10(62.5)$ & $19(70.4)$ & 0.7 \\
\hline Prosthetic valve endocarditis & $14(32.5)$ & $6(37.5)$ & $8(29.6)$ & 0.72 \\
\hline \multicolumn{5}{|l|}{ Heart valve affected } \\
\hline Aortic & $31(72.1)$ & $11(68.8)$ & $20(74.1)$ & 0.69 \\
\hline Mitral & $12(27.9)$ & $2(12.5)$ & $10(37)$ & 0.15 \\
\hline Right-sided IE & $2(4.6)$ & $1(6.2)$ & $1(3.7)$ & 1.0 \\
\hline Complications $^{\mathrm{a}}$ & $6(13.9)$ & $3(18.8)$ & $3(11.1)$ & 0.5 \\
\hline Surgery performed & $18(41.8)$ & $7(43.7)$ & $11(40.7)$ & 0.9 \\
\hline Duration of treatment & $52 \pm 22.6$ & $45 \pm 21.1$ & $56 \pm 23.2$ & 0.02 \\
\hline 30-Day mortality rate & $7(16.2)$ & $1(6.2)$ & $6(22.2)$ & 0.41 \\
\hline Failure at the end of treatment & $2(4.6)$ & $1(6.2)$ & $1(3.7)$ & 1.0 \\
\hline
\end{tabular}

Bold value indicates statistical significance $(p<0.05)$

$D B R$ daptomycin-containing regimen, $D S R$ daptomycin-sparing regimen, $I E$ infective endocarditis

${ }^{a}$ Complications: heart failure, new renal failure, embolism, paravalvular complications, stroke

\section{RESULTS}

\section{General Characteristics of the Cohort and Strains}

During the 8-year study period, enterococcal $I E$ accounted for 43 of 405 (10.6\%) IE episodes, including 29 NVE, (67.4\%) and 14 PVE (32.6\%). The median age of the cohort was 67 years, and $29(67.4 \%)$ patients were male. The main comorbidities are shown in Table 1 . There was no statistically significant difference between groups regarding baseline clinical characteristics, except for diabetes.

Enterococcus faecalis accounted for 39 episodes $(90.7 \%)$, while E. faecium for 4 (9.3\%). All strains treated with daptomycin were susceptible (MICs ranged from 0.25 to $4 \mathrm{mg} / \mathrm{l}$ ), with 13 strains with MIC $\leq 1 \mathrm{mg} / \mathrm{l}$ and 3 strains with MIC $2-4 \mathrm{mg} / \mathrm{l}$. Thirteen Enterococcus strains 


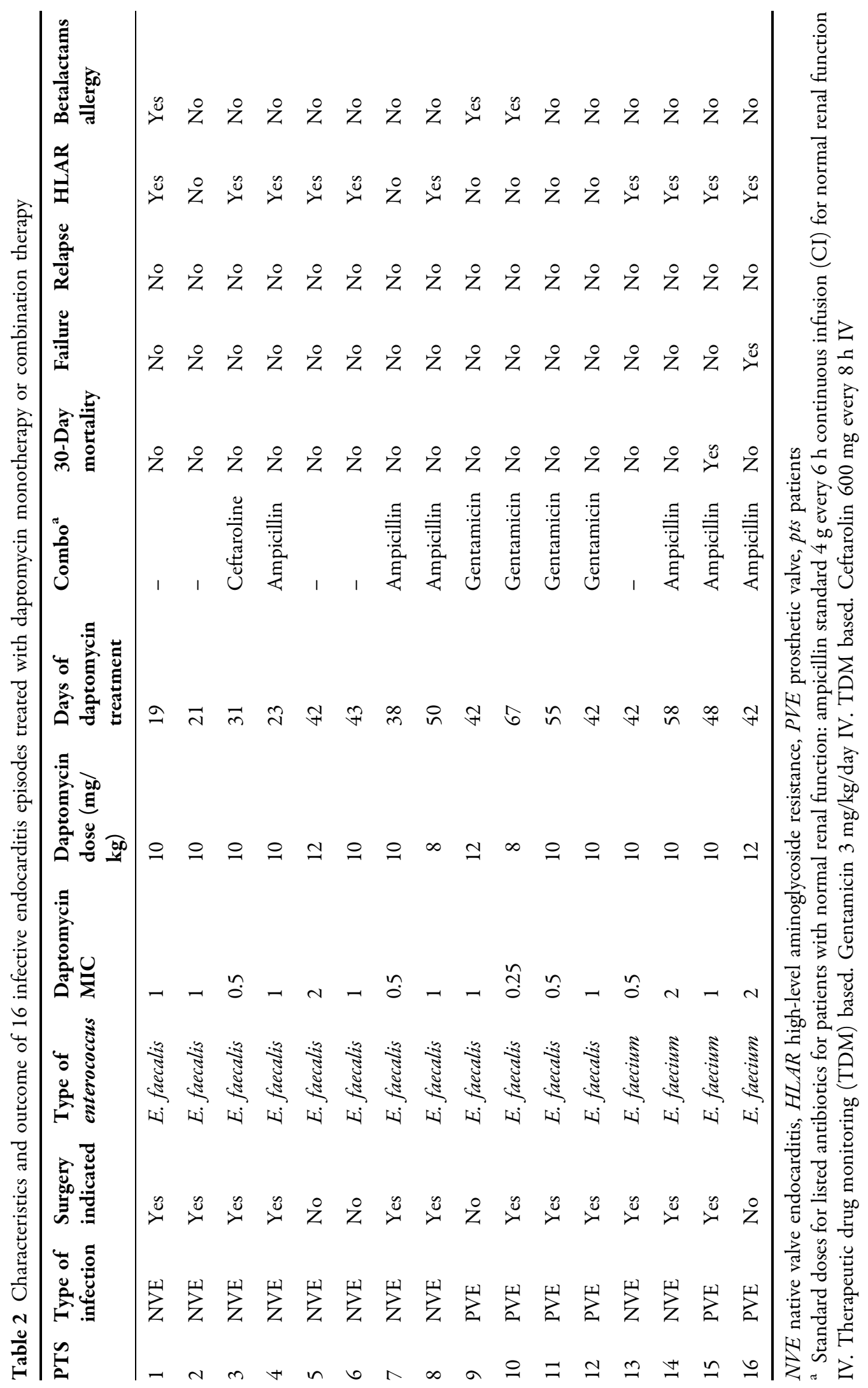


showed HLAR (12 E. faecalis and 1 E. faecium) (Table 2).

\section{Use of Daptomycin, Outcome and Comparison}

Of the 43 patients included in the study, 16 (37.2\%) were treated with DBR, 14 (32.5\%) were ampicillin/gentamicin-treated patients, 9 (20.9\%) were ampicillin/ceftriaxone-treated patients, and the rest were patients with alternative regimens ( 2 with vancomycin, 1 with linezolid, 1 with levofloxacin). Three of 16 patients in the DBR group (18.7\%) had a history of beta-lactam allergy. In the DBR group, daptomycin combination therapy was performed in 11 patients $(68.7 \%)$, in 6 cases $(54.5 \%)$ in association with ampicillin, in 4 cases $(36.5 \%)$ with gentamicin and in the remaining case (9\%) with ceftaroline. The mean administered dose of daptomycin was $10.125 \mathrm{mg} / \mathrm{kg} /$ day intravenously (range $8-12 \mathrm{mg} / \mathrm{kg} /$ day). Mean length of antimicrobial therapy was 45 days in the DBR group and 56 days in the DSR $(P=0.02)$. No related adverse events (rhabdomyolysis and eosinophilic pneumonia) occurred in patients undergoing treatment with high-dose daptomycin.

Overall surgery was performed in $41.8 \%$ of patients (Tables 1, 2). All-cause mortality at 30 days was reported in seven (16.2\%) patients. Overall, patients treated with DBR compared with patients treated with DSR had no higher mortality rates and/or failure at end of therapy (6.2\% vs. $22.2 \% ; P=0.41$ and MICs $0.25-2 \mathrm{mg} / \mathrm{l}$ $6.2 \%$ vs. $3.7 \% ; P=1.0$ ). In the sub-group of patients with NVE and PVE treated with DBR and DSR, no difference was found regarding the primary endpoints on the single or combined use of daptomycin (Table 2). There was only one relapse in the DBR group.

\section{DISCUSSION}

To the best of our knowledge, this is the largest series of enterococcal endocarditis patients treated with a high-dose daptomycin regimen, suggesting that it could be an alternative treatment option for enterococcal NVE and PVE.
Our results are in contrast with previous retrospective studies that found worse microbiologic and clinical response in patients with EIE treated with DBR [12]. Enterococcus spp. typically exhibit a higher in vitro MIC to daptomycin than $S$. aureus, with a threshold for susceptibility that is four times as high $(\leq 4$ vs. $\leq 1 \mathrm{mg} / \mathrm{l}$ for $S$. aureus), and daptomycin non-susceptibility among enterococci, currently defined as MIC $>4 \mathrm{mg} / \mathrm{l}$, is a growing concern [16]. In our study, all strains treated with daptomycin were susceptible, with 13 strains with $\mathrm{MIC} \leq 1 \mathrm{mg} / \mathrm{l}$ and 3 strains with MIC 2-4 mg/l. Actually current CLSI guidelines have proposed new breakpoints, suggesting that a breakpoint $\leq 1 \mu \mathrm{g} / \mathrm{ml}$ is likely more predictive of "true" susceptibility, particularly in endovascular infections. In addition, for susceptible-dose dependent strains (MIC 2-4 $\mu \mathrm{g} / \mathrm{Ml}$ ), increased daptomycin doses (10-12 $\mathrm{mg} / \mathrm{kg} /$ day) are recommended with potential consideration for combination therapy [17].

In keeping with our center experience and previous studies, to overcome this issue, two approaches can be performed. On the one hand, the use of higher dosing and then the use of daptomycin combination therapy could influence the efficacy of daptomycin and also prevent development of resistance leading to both clinical and microbiologic failures $[7,15]$. In our study, most patients were treated with high daptomycin doses (mean $10.125 \mathrm{mg} / \mathrm{kg} /$ day). Probably the delayed response observed in previous studies on EIE could be explained by the lower doses used (average dose of $8.5 \mathrm{mg}$ / $\mathrm{kg} /$ day) [12], since the use of increased dosage has reported higher and more sustained killing, including for E. faecium [14, 15, 18, 19].

In our series, most patients received combination therapy $(68.7 \%)$, mainly in association with ß-lactam $(7,43.7 \%)$ followed by aminoglycoside $(4,25 \%)$. Several in vitro studies have shown that daptomycin combination with $ß$ lactam agents such as ampicillin, ceftriaxone and ceftaroline/ceftobiprole could result in a synergistic effect due to increased binding to the cell membrane target $[11,20]$. However, the co-administration of gentamicin has not shown a meaningful effect on the pharmacokinetic or bactericidal activity of daptomycin on 
Enterococcus spp. [21]. Of note, the emergence of resistance has been shown to be delayed by $ß-$ lactam, but not by other antibiotics (gentamicin and rifampin) [7].

Moreover, daptomycin is not altered in biofilm bacteria in the stationary growth phase, which makes it a major agent for the treatment of EIE, including PVE [15]. Of note, in line with previous literature, in our series, no patient experienced daptomycin-induced muscle toxicity or eosinophilic pneumonia, despite the high dosing regimen [22].

In our cohort, patients treated with DBR compared with patients treated with DSR had a trend toward lower mortality and a significantly shorter treatment duration (45 days in the DBR group and 56 days in the DSR; $P=0.02$ ). Treatment of EIE is known to be difficult, and medical management is a challenge. In patients undergoing heart surgery for IE, the optimal length of antibiotic therapy after surgical excision is an unresolved issue. Current European guidelines recommend administering the complete course of treatment if the resected tissue is culture-positive and completion of the prescheduled course if it is culture-negative [23]. In our center, we have a standardized protocol for EIE. Patients with EIE receive a complete 6-week course of treatment. The duration of treatment is individualized according to the complications of IE and the result of valve culture. In patients who have undergone valve replacement for IE, if valve culture is negative, a 2-week postsurgical treatment course in usually performed, and if the valve culture is positive, a 4 -week postsurgical treatment course is usually performed.

The main limitations of this study are its single-center design and the small sample size.

Second, a multivariate regression analysis would have offered robust support, but we found a statistical limitation related to the small size of the cohort.

However, as far as we know, this is the largest prospective series of EIE treated with high-dose daptomycin. The sampling approach for selecting subjects for DBR was not random and was subjective, but was performed by clinicians involved in daily practice in EIE management and reflects real-life practice in our center.

\section{CONCLUSION}

In conclusion, our findings suggest that highdosing daptomycin regimens could be used as an alternative treatment option in enterococcal NVE and PVE even in monotherapy. This strategy deserves to be studied in prospective and controlled clinical trials.

\section{ACKNOWLEDGMENTS}

The authors acknowledge the clinical staff and patients of University of Udine and Azienda Sanitaria Universitaria Integrata di Udine.

Funding. No funding or sponsorship was received for this study or publication of this article.

Authorship. All named authors meet the International Committee of Medical Journal Editors (ICMJE) criteria for authorship for this article, take responsibility for the integrity of the work as a whole, and have given their approval for this version to be published.

Prior Presentation. This work was presented as an abstract with poster presentation at ECCMID 2019, Amsterdam.

Disclosures. Alessandro Russo, Filippo Givone, Martina Ingani and Elena Graziano have nothing to disclose. Outside the submitted work, Matteo Bassetti has received funding for scientific advisory boards, travel and speaker honoraria from Angelini, AstraZeneca, Bayer, Cubist, Pfizer, Melinta Therapeutics, Menarini, MSD, Nabriva, Paratek, Roche, Shionogi, Tetraphase, The Medicines Company and Astellas Pharma Inc. Matteo Bassetti is also a member of the journal's Editorial Board. Outside the submitted work, Maddalena Peghin has received funding for speaker honoraria from Dia Sorin, Thermo Fisher and Pfizer.

Compliance with Ethics Guidelines. All procedures performed in studies involving human participants were in accordance with 
the ethical standards of University of Udine and Azienda Sanitaria Universitaria Integrata di Udine (IRB NR 505) and with the 1964 Helsinki Declaration and its later amendments or comparable ethical standards. The need for informed consent was waived by the IRB because of the observational nature and the minimal risk of the study.

Data Availability. The data sets generated during and/or analyzed during the current study are not publicly available because of privacy concerns, but are available from the corresponding author on reasonable request.

Open Access. This article is distributed under the terms of the Creative Commons Attribution-NonCommercial 4.0 International License (http://creativecommons.org/licenses/ by-nc/4.0/), which permits any noncommercial use, distribution, and reproduction in any medium, provided you give appropriate credit to the original author(s) and the source, provide a link to the Creative Commons license, and indicate if changes were made.

\section{REFERENCES}

1. Selton-Suty C, Celard M, Le Moing V, Doco-Lecompte T, Chirouze C, Iung B, Strady C, Revest M, Vandenesch F, Bouvet A, et al. Preeminence of Staphylococcus aureus in infective endocarditis: a 1-year population-based survey. Clin Infect Dis. 2012;54(9):1230-9.

2. Munoz P, Kestler M, De Alarcon A, Miro JM, Bermejo J, Rodriguez-Abella $\mathrm{H}$, Farinas MC, Cobo Belaustegui M, Mestres C, Llinares P, et al. Current epidemiology and outcome of infective endocarditis: a multicenter, prospective, cohort study. Medicine. 2015;94(43):e1816.

3. Habib G, Lancellotti P, Antunes MJ, Bongiorni MG, Casalta JP, DelZotti F, Dulgheru R, El Khoury G, Erba PA, Iung B, et al. 2015 ESC Guidelines for the management of infective endocarditis: the task force for the management of infective endocarditis of the European Society of Cardiology (ESC). Endorsed by: European Association for CardioThoracic Surgery (EACTS), the European Association of Nuclear Medicine (EANM). Eur Heart J. 2015;36(44):3075-128.
4. Fernandez-Hidalgo N, Almirante B, Gavalda J, Gurgui M, Pena C, de Alarcon A, Ruiz J, Vilacosta I, Montejo M, Vallejo N, et al. Ampicillin plus ceftriaxone is as effective as ampicillin plus gentamicin for treating enterococcus faecalis infective endocarditis. Clin Infect Dis. 2013;56(9):1261-8.

5. Escola-Verge L, Fernandez-Hidalgo N, RodriguezPardo D, Pigrau C, Gonzalez-Lopez JJ, Bartolome R, Almirante B. Teicoplanin for treating enterococcal infective endocarditis: a retrospective observational study from a referral centre in Spain. Int J Antimicrob Agents. 2019;53(2):165-70.

6. Tobudic S, Forstner C, Burgmann $H$, Lagler $H$, Ramharter M, Steininger C, Vossen MG, Winkler S, Thalhammer F. Dalbavancin as primary and sequential treatment for gram-positive infective endocarditis: 2-year experience at the general hospital of vienna. Clin Infect Dis. 2018;67(5):795-8.

7. Nigo M, Munita JM, Arias CA, Murray BE. What's new in the treatment of enterococcal endocarditis? Curr Infect Dis Rep. 2014;16(10):431.

8. Humphries RM, Pollett S, Sakoulas G. A current perspective on daptomycin for the clinical microbiologist. Clin Microbiol Rev. 2013;26(4):759-80.

9. Fowler VG Jr, Boucher HW, Corey GR, Abrutyn E, Karchmer AW, Rupp ME, Levine DP, Chambers HF, Tally FP, Vigliani GA, et al. Daptomycin versus standard therapy for bacteremia and endocarditis caused by Staphylococcus aureus. N Engl J Med. 2006;355(7):653-65.

10. Smith JR, Barber KE, Raut A, Rybak MJ. beta-Lactams enhance daptomycin activity against vancomycin-resistant Enterococcus faecalis and Enterococcus faecium in in vitro pharmacokinetic/ pharmacodynamic models. Antimicrob Agents Chemother. 2015;59(5):2842-8.

11. Pericas JM, Garcia-de-la-Maria C, Brunet M, Armero Y, Garcia-Gonzalez J, Casals G, Almela M, Quintana E, Falces C, Ninot S, et al. Early in vitro development of daptomycin non-susceptibility in highlevel aminoglycoside-resistant Enterococcus faecalis predicts the efficacy of the combination of highdose daptomycin plus ampicillin in an in vivo model of experimental endocarditis. J Antimicrob Chemother. 2017;72(6):1714-22.

12. Ceron I, Munoz P, Marin M, Segado A, Roda J, Valerio M, Bouza E. Group for the management of infective endocarditis of the Gregorio Maranon $\mathrm{H}$ : efficacy of daptomycin in the treatment of enterococcal endocarditis: a 5 year comparison with conventional therapy. J Antimicrob Chemother. 2014;69(6):1669-74. 
13. Carugati M, Bayer AS, Miro JM, Park LP, Guimaraes AC, Skoutelis A, Fortes CQ, Durante-Mangoni E, Hannan MM, Nacinovich F, et al. High-dose daptomycin therapy for left-sided infective endocarditis: a prospective study from the international collaboration on endocarditis. Antimicrob Agents Chemother. 2013;57(12):6213-22.

14. Lubbert C, Rodloff AC, Hamed K. Real-world treatment of enterococcal infections with daptomycin: insights from a large European registry (EU-CORE). Infect Dis Therapy. 2015;4(3):259-71.

15. Senneville E, Caillon J, Calvet B, Jehl F. Towards a definition of daptomycin optimal dose: lessons learned from experimental and clinical data. Int J Antimicrob Agents. 2016;47(1):12-9.

16. Douglas AP, Marshall C, Baines SL, Ritchie D, Szer J, Madigan V, Chan HT, Ballard SA, Howden BP, Buising $\mathrm{K}$, et al. Utilizing genomic analyses to investigate the first outbreak of vanA vancomycinresistant Enterococcus in Australia with emergence of daptomycin non-susceptibility. J Med Microbiol. 2019;68(3):303-8.

17. https://clsi.org/.

18. Hall AD, Steed ME, Arias CA, Murray BE, Rybak MJ. Evaluation of standard- and high-dose daptomycin versus linezolid against vancomycin-resistant Enterococcus isolates in an in vitro pharmacokinetic/ pharmacodynamic model with simulated endocardial vegetations. Antimicrob Agents Chemother. 2012;56(6):3174-80.

19. Chuang YC, Chen PY, Lin CY, Chen YC, Wang JT, Chang SC. A retrospective clinical comparison of daptomycin vs daptomycin and a beta-lactam antibiotic for treating vancomycin-resistant Enterococcus faecium bloodstream infections. Sci Rep. 2018;8(1):1632.

20. Sakoulas G, Bayer AS, Pogliano J, Tsuji BT, Yang SJ, Mishra NN, Nizet V, Yeaman MR, Moise PA. Ampicillin enhances daptomycin- and cationic host defense peptide-mediated killing of ampicillinand vancomycin-resistant Enterococcus faecium. Antimicrob Agents Chemother. 2012;56(2):838-44.

21. DeRyke CA, Sutherland C, Zhang B, Nicolau DP, Kuti JL. Serum bactericidal activities of high-dose daptomycin with and without coadministration of gentamicin against isolates of Staphylococcus aureus and Enterococcus species. Antimicrob Agents Chemother. 2006;50(11):3529-34.

22. Benvenuto M, Benziger DP, Yankelev S, Vigliani G. Pharmacokinetics and tolerability of daptomycin at doses up to 12 milligrams per kilogram of body weight once daily in healthy volunteers. Antimicrob Agents Chemother. 2006;50(10):3245-9.

23. Habib G, Hoen B, Tornos P, Thuny F, Prendergast B, Vilacosta I, Moreillon P, de Jesus AM, Thilen U, Lekakis J, et al. Guidelines on the prevention, diagnosis, and treatment of infective endocarditis (new version 2009): the Task Force on the Prevention, Diagnosis, and Treatment of Infective Endocarditis of the European Society of Cardiology (ESC). Endorsed by the European Society of Clinical Microbiology and Infectious Diseases (ESCMID) and the International Society of Chemotherapy (ISC) for Infection and Cancer. Eur Heart J. 2009;30(19):2369-413. 